\title{
Segregação alimentar entre duas espécies de Heptapteridae no Rio Macaé, RJ
}

\author{
Clarissa Brazil-Sousa ${ }^{1,2}$, Raquel Monteiro Marques ${ }^{1}$ \& Míriam Pilz Albrecht ${ }^{1}$ \\ ${ }^{1}$ Laboratório de Ecologia de Peixes, Departamento de Ecologia, Instituto de Biologia - IB, \\ Centro de Ciências da Saúde - CCS, Universidade Federal do Rio de Janeiro - UFRJ, \\ Av. Pau Brasil, 211, CEP 21941-590, Ilha do Fundão, Rio de Janeiro, RJ, Brasil \\ ${ }^{2}$ Autor para correspondência: Clarissa Brazil-Sousa,e-mail: brazil_sousa@yahoo.com.br
}

BRAZIL-SOUSA, C., MARQUES, R.M. \& ALBRECHT, M.P. Food partitioning between two heptapterid fish species in Macaé River, RJ (Southeastern Brazil). Biota Neotrop. 9(3): http://www.biotaneotropica.org. br/v9n3/en/abstract?article+bn00309032009.

\begin{abstract}
Food is the main axis of niche partitioning among fishes, being an important factor for the organization of their populations. We herein investigate this premise for two closely related species, by characterization and comparison of the natural diet of two heptapterid fishes, Rhamdioglanis transfasciatus and Rhamdia quelen along the Macaé River, Rio de Janeiro state, Southeastern Brazil. R. quelen was classified as carnivore, and the most important items of its diet were crustaceans and fishes. $R$. transfasciatus was classified as invertivore, as it ate mainly trichopteran and dipteran larvae. The calculation of the trophic position algorithm showed that both species are secondary consumers, but R. quelen had a slightly higher value. Both species can be considered as generalists but with differences of feeding strategies: some individuals of $R$. quelen behave as specialists, whereas most individuals of $R$. transfasciatus population act as generalists. Intraspecific comparisons revealed a similar diet of each species between the two sampling sites, however, the niche overlap between the populations of the two species was very low in the sites where both occurred, indicating the existence of resource partitioning between Rhamdia quelen e Rhamdioglanis transfasciatus in Macaé river.

Keywords: feeding, niche, resource partitioning, Rhamdioglanis transfasciatus, Rhamdia quele.
\end{abstract}

BRAZIL-SOUSA, C., MARQUES, R.M. \& ALBRECHT, M.P. Segregação alimentar entre duas espécies de Heptapteridae no Rio Macaé, RJ. Biota Neotrop. 9(3): http://www.biotaneotropica.org.br/v9n3/pt/ abstract?article+bn00309032009.

Resumo: Alimento é considerado o principal eixo da partilha de nicho entre peixes, sendo um fator importante para a organização de suas populações. Com o intuito de investigar essa assertiva para duas espécies filogeneticamente próximas, a dieta natural de dois peixes da família Heptapteridae, Rhamdioglanis transfasciatus e Rhamdia quelen foi caracterizada e comparada ao longo do curso longitudinal do rio Macaé. $R$. quelen foi classificada como carnívora, sendo crustáceos e peixes os itens mais importantes da sua dieta, ao passo que $R$. transfasciatus foi considerada invertívora, tendo consumido principalmente larvas de Trichoptera e de Diptera. A posição trófica calculada revelou que ambas as espécies são consumidoras secundárias, com valores um pouco maiores para $R$. quelen. As espécies podem ser consideradas generalistas, mas com algumas diferenças na estratégia alimentar adotada por suas populações: alguns grupos de indivíduos de $R$. quelen se comportaram de forma especialista, enquanto que a população de $R$. transfasciatus se comporta de forma generalista em sua maioria. Na comparação intraespecífica, a dieta de ambas foi muito semelhante entre as duas localidades, porém a sobreposição de nicho entre as populações das duas espécies foi quase nula nos locais de coocorrência, indicando a existência de partilha alimentar entre Rhamdia quelen e Rhamdioglanis transfasciatus no rio Macaé.

Palavras-chave: alimentação, nicho, partilha de recursos, Rhamdioglanis transfasciatus, Rhamdia quelen. 


\section{Introdução}

A partilha de recursos entre espécies é considerada como um dos processos responsáveis pela estruturação de comunidades (Schoener 1974). Considerando o conceito n-dimensional de nicho (sensu Hutchinson 1957), essa partilha pode se dar ao longo de vários eixos, sendo fruto de diversos fatores (Ross 1986). Em vertebrados, a segregação ocorre principalmente ao longo dos eixos Alimento e Espaço (Schoener 1974), sendo que, em peixes, alimento é a principal causa de partilha de nicho (Ross 1986), consistindo um fator importante para a organização das populações desses organismos.

Espécies simpátricas com poucas diferenças anatômicas apresentam forte potencial competitivo (Wootton 1990), sendo esperadas variações ecológicas como utilização distinta de hábitats e/ou partilha de recursos para coexistirem (Connel 1978, Herder \& Freyhof 2006). Quando os recursos alimentares são suficientes para manter duas ou mais populações pode haver sobreposição, geralmente parcial, do nicho (Pianka 1973), sendo possível manter essa situação sem prejuízo para ambas. Entretanto, quando o recurso é escasso, há tendência, nos peixes, a modificar suas dietas, através do uso de recursos alternativos (e.g. Fugi \& Hahn 1991, Andrian et al. 1994, Almeida 1994, Andrian \& Barbieri 1996, Loureiro \& Hahn 1996). Em peixes neotropicais, essa plasticidade trófica é apresentada por grande parte das espécies (Weatherley 1963, Lowe-McConnell 1987, Abelha et al. 2001). Entretanto, espera-se que restrições morfológicas (e.g. tamanho da boca) condicionem a amplitude do que pode ser ou não consumido pela espécie, pois a capacidade de explorar os diferentes recursos depende da adequação a sua tática alimentar, aparato alimentar e capacidade digestiva (Gerking 1994, Wootton 1990).

A família Heptapteridae representa uma das maiores radiações de bagres neotropicais, sendo composta por aproximadamente 150-200 espécies válidas, distribuídas do sul da Argentina ao norte do México (Bockmann \& Guazzelli 2003). Devido à morfologia semelhante, são esperados hábitos semelhantes para os peixes da família Heptapteridae (Bockmann \& Guazzelli 2003). No rio Macaé, situado na região biogeográfica das Bacias do Leste, no norte fluminense, foram encontradas quatro espécies dessa família: Rhamdia quelen (Quoy \& Gaimard 1824), Rhamdioglanis transfasciatus MirandaRibeiro, 1908, Acentronichthys leptos Eigenmann \& Eigenmann, 1889 e Pimelodella lateristriga (Lichtenstein 1823) (Brito 2007). Rhamdia quelen se distribui do sul do México ao rio da Prata e $R$. transfasciatus possui distribuição restrita a riachos costeiros entre os estados do Rio de Janeiro e Santa Catarina (Menezes et al. 2007). Acentronichthys leptos e P. lateristriga não foram incluídas nesse estudo devido à sua baixa abundância e distribuição restrita na área de estudo, ocorrendo somente em algumas localidades do rio. Rhamdia quelen e $R$. transfasciatus foram capturadas ao longo do rio, nas altitudes de 1020 a 10 m tendo sido encontradas em maior abundância no começo do trecho médio superior, entre 670 e $610 \mathrm{~m}$ de altitude. Nesses pontos, as espécies co-ocorreram, entretanto, $R$. transfasciatus foi predominantemente capturada com rede de espera e kicking (deslocamento de pedras em frente à rede manual) no canal e $R$. quelen com redes de espera em remansos (Brito 2007). Há poucos registros sobre a biologia em ambiente natural da família Heptapteridae e isso se reflete no conhecimento produzido sobre as duas espécies. Estudos sobre a biologia de $R$. quelen são principalmente voltados para seu potencial para piscicultura, uma vez que apresenta grande aceitação pelo mercado consumidor (e.g. Gomes et al. 2000, Carneiro \& Mikos 2005), ao passo que não foram encontrados registros na literatura sobre a biologia de $R$. transfasciatus.

A determinação da dieta natural, assim como dos aspectos associados à alimentação, tem sido apontada como essencial para o entendimento acerca da ecologia das populações de peixes (Micheletti
\& Uieda 1996) e seu papel nos processos que regulam os ecossistemas (Resetarits \& Chalcraft 2007). Vários estudos teóricos recentes têm usado o conceito de nicho para o entendimento do papel da diversidade nos processos ecossistêmicos (e.g. Loreau 2000). Em pequenas escalas, espécies relacionadas que co-ocorrem em comunidades locais geralmente exibem diferenciações temporais ou espaciais de micro-hábitat, uso de recursos, dieta ou outros fatores. Assim, partindo da hipótese de que há alguma diferenciação ecológica que permita a coexistência de Rhamdioglanis transfasciatus e de Rhamdia quelen, objetivamos descrever a dieta natural dessas espécies e compará-las nos locais de coocorrência no curso longitudinal do rio Macaé.

\section{Material e Métodos}

\section{1. Área de estudo}

O rio Macaé $\left(22^{\circ} 21^{\prime}-22^{\circ} 28^{\prime} \mathrm{S}\right.$ e $\left.42^{\circ} 27^{\prime}-42^{\circ} 35^{\prime} \mathrm{W}\right)$ é um rio de tamanho médio com cerca de $130 \mathrm{~km}$ de extensão localizado no Norte Fluminense. A bacia do rio Macaé, inserida na região biogeográfica das Bacias do Leste, é uma das principais do Estado do Rio de Janeiro. O rio nasce a $1500 \mathrm{~m}$ de altitude e deságua no Oceano Atlântico, junto à cidade de Macaé, como um rio de sexta ordem (SEMADS 2001, Baptista et al. 2001, Amaral 2003) . Apresenta grande variedade de ambientes em seu entorno devido aos diferentes usos do solo que variam ao longo do rio, podendo compreender desde extensas áreas de Mata Atlântica bem conservada (no alto curso) até grandes áreas de pastagens (como no baixo curso) (Bizerril \& Primo 2001). O canal é pedregoso em todo o trecho ritral do rio e se torna arenoso no potamal, com eventuais pedras aluviais.

\section{Amostragens}

Os espécimes de $R$. quelen e $R$. transfasciatus estudados foram capturados entre março de 2004 e março de 2005 em 10 dos 12 pontos de coleta distribuídos ao longo de quatro trechos do rio Macaé (Figura 1): superior (pontos RM1 a RM3), médio superior (RM4 a RM6), médio inferior (RM7 e RM8) e inferior (RM9 a RM12) (Brito 2007).

Os exemplares foram capturados com redes de espera de $10 \mathrm{~m}$ de comprimento e 1,5 de altura com malhas de 15, 20, 25, 30, 35, 40 e $50 \mathrm{~mm}$ entre nós adjacentes, armadas ao fim da tarde e recolhidas na manhã seguinte. Foram também utilizados anzóis com iscas artificiais, tarrafas, picaré, peneira e arrasto com malhas de 25 e $30 \mathrm{~mm}$ entre nós adjacentes (Brito 2007). Os peixes foram fixados em formol 10\% e conservados em álcool 70\%. Em laboratório foram dissecados para obtenção de dados biológicos e retirada do trato digestório.

Foi analisado o conteúdo estomacal de 41 espécimes de $R$. quelen (comprimento padrão: $21,5 \mathrm{~cm} \pm 5,6)$ e 70 de $R$. transfasciatus (comprimento padrão: $10,3 \mathrm{~cm} \pm 3,9$ ). Os itens alimentares foram identificados até o nível taxonômico mais baixo possível com auxílio de literatura especializada (Borror \& Delong 1969, Merrit \& Cummins 1996) e quantificados quanto ao volume e à frequência de ocorrência (Hyslop 1980, Hynes 1950, Cortés 1997), sendo o volume obtido através de uma placa de vidro quadrada $(10 \mathrm{~cm}$ de lado) com bordas de $1 \mathrm{~mm}$ de altura e uma escala milimetrada colocada embaixo, conforme descrito em Albrecht \& Caramaschi (2003). Posteriormente, seus valores foram combinados em um índice alimentar (IAi) de acordo com Kawakami \& Vazzoler (1980).

A posição trófica (TP) de cada espécie de peixe foi calculada através do algoritmo de Adams et al. (1983). Material inorgânico, produtores primários (algas e plantas vasculares) e detritos foram considerados como $\mathrm{TP}=0,0$ e consumidores primários como $\mathrm{TP}=1,0$. A posição trófica de invertebrados (presas) foi estimada a partir de dados da literatura (Borror et al. 1992, Merrit \& Cummins 


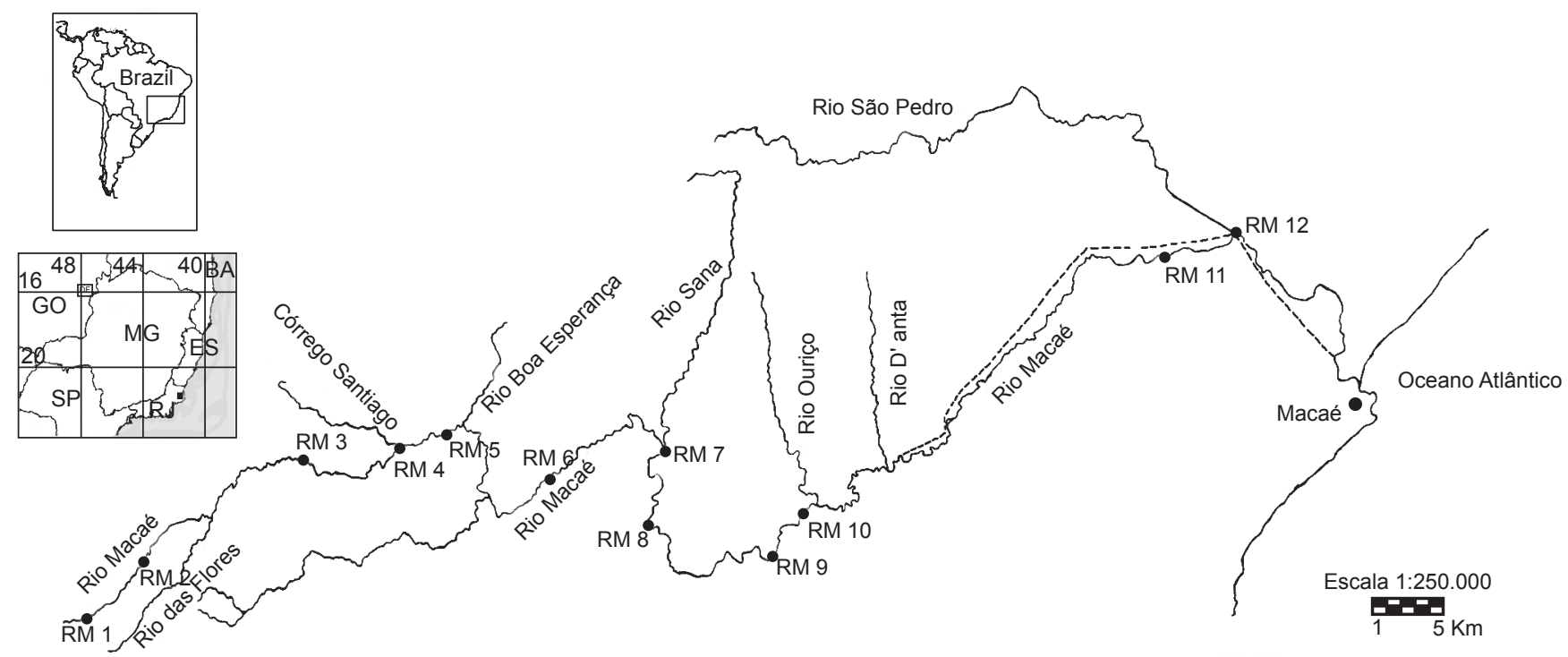

Figura 1. Localização geográfica do rio Macaé e pontos de coleta ao longo da área de estudo. Fonte: Brito (2007).

Figure 1. Geographic location of Macaé River and sampling sites along the study area. Source: Brito (2007).

1996, Albrecht 2005) sobre a alimentação de cada grupo taxonômico (formas imaturas ou adultas de cada família ou ordem).

A razão entre o comprimento do intestino e o comprimento padrão dos exemplares coletados foi calculada a fim de obter o quociente intestinal médio (Qi) das populações, sendo relacionados posteriormente com a dieta das espécies. A relação entre o Qi e a dieta refere-se ao processo adaptativo de encurtamento do intestino na medida em que há uma substituição de dieta baseada em vegetais por outra, baseada em itens animais. Em uma mesma classe de tamanho, o comprimento do intestino dos herbívoros é maior do que o de onívoros e esses, por sua vez, possuem o intestino mais longo do que o dos carnívoros (Kramer \& Bryant 1995).

A amplitude de nicho foi calculada através do Índice de Diversidade de Shannon (Krebs 1999) e comparada estatisticamente pelo teste de Hutcheson (Zar 1984). As estratégias alimentares das espécies foram analisadas por meio do método gráfico modificado de Amundsen et al. (1996). A partir desse método, informações sobre a ecologia alimentar das espécies são obtidas através da relação gráfica entre abundância presa-específica ( $\mathrm{P} i$ ) e sua frequência de ocorrência (Fi), cujos cálculos estão demonstrados abaixo (Equações 1 e 2).

$\% \mathrm{Pi}=(\Sigma \mathrm{Si} / \Sigma \mathrm{Sti}) \times 100$

onde, $\mathrm{Si}=$ número de estômagos que contêm apenas a presa i; $\mathrm{Sti}=$ total de estômagos em que a presa $\mathrm{i}$ ocorre.

$\% \mathrm{Fi}=(\mathrm{Ni} / \mathrm{N}) \times 100$

onde, $\mathrm{Ni}$ = número de indivíduos com a presa i no estômago; $\mathrm{N}=$ total de indivíduos com conteúdo estomacal.

Informações sobre a importância da presa e a estratégia alimentar do predador são obtidos a partir da análise da distribuição dos pontos ao longo dos eixos do gráfico. Para essa análise, os itens alimentares foram agrupados em categorias mais amplas, para melhor visualização dos resultados.

Como medida de similaridade foi utilizado o índice de Pianka (1973) para comparação da dieta entre as espécies nos locais de coocorrência e dentro da mesma espécie em diferentes locais. Os valores variam de 0 a 1 , sendo 0 indicador de segregação alimentar total e 1 de sobreposição total na dieta.

\section{Resultados}

\section{Caracterização da alimentação natural}

Na dieta de R. quelen, Crustáceos e Peixes (IAi = 47,85 e 42,83\%, respectivamente) foram os itens mais importantes e na dieta de R. transfasciatus, foram Larvas de Trichoptera (48,95\%) e de Diptera $(35,50 \%)$ (Tabela 1). Rhamdia quelen atingiu maior posição trófica calculada (PT), assim como maior quociente intestinal (Qi). Foi encontrada uma riqueza de 53 itens alimentares na dieta de $R$. quelen e 43 na de $R$. transfasciatus. $R$. quelen apresentou a maior amplitude de nicho (H'). H' foi significativamente diferente entre as espécies $(\mathrm{p}<0,05)$. O grau de sobreposição de nicho $(\theta)$ entre as espécies foi 0,51 . Os valores de S, H', PT, Qi e $\theta$ para ambas as espécies, considerando todos os indivíduos analisados, estão relacionados na Tabela 2.

O método gráfico de Amundsen et al. (1996) revelou diferenças na estratégia alimentar adotada por suas populações. Na Figura 2a é possível observar que a população analisada de $R$. quelen apresenta grupos de indivíduos que se comportam de forma especialista em relação a alguns itens consumidos em grandes quantidades. Apenas Material Vegetal foi consumido por mais de $50 \%$ da população, mas em quantidades muito baixas. Já para R. transfasciatus (Figura 2b) pode-se observar que a maior parte dos indivíduos da população são generalistas, apresentando consumo dos itens em pequenas quantidades por menos da metade dos indivíduos. Apenas Larva de Diptera e de Trichoptera foram consumidos por mais de $50 \%$ da população, coincidindo com os itens que obtiveram maior valor de IAi.

\section{Comparação nos locais de coocorrência}

Na comparação intraespecífica, a dieta de ambas as espécies foi muito semelhante entre as duas localidades $(\theta=0,98$ para $R$. quelen e $\theta=0,91$ para $R$. transfasciatus). Na comparação interespecífica, o grau de sobreposição de nicho entre as populações foi baixo em ambos os locais de coocorrência (Tabela 3). Em RM4 R. quelen apresentou uma riqueza menor dos itens consumidos, mas em RM5 apresentou maior riqueza de itens, em relação a $R$. transfasciatus. Entretanto, a amplitude do nicho alimentar ( $\left.\mathrm{H}^{\prime}\right)$ nos locais de coocorrência não 
Tabela 1. Índices de importância (IAi) dos itens alimentares consumidos por Rhamdia quelen (Rq) e Rhamdioglanis transfasciatus (Rt) ao longo do rio Macaé, RJ. As letras que sucedem as ordens dos artrópodos denotam seu estágio de desenvolvimento, sendo: $\mathrm{A}=$ adultos; $\mathrm{L}=$ larvas; $\mathrm{N}=$ ninfas e $\mathrm{P}=$ pupas.

Table 1. Importance indices (IAi) of food items consumed by Rhamdia quelen (Rq) and Rhamdioglanis transfasciatus (Rt) along the Macaé River, RJ. Letters following arthropods orders demonstrate their developmental stage: $\mathrm{A}=$ adults; $\mathrm{L}=$ larvae; $\mathrm{N}=$ nymphs and $\mathrm{P}=$ pupae.

\begin{tabular}{|c|c|c|}
\hline Itens alimentares & $\mathbf{R q}$ & $\mathbf{R t}$ \\
\hline Alga filamentosa & 0,003 & - \\
\hline Aracnidae & 0,024 & 0,198 \\
\hline Caelifera & 0,253 & - \\
\hline Coleoptera & 0,379 & 2,884 \\
\hline Decapoda & 47,849 & - \\
\hline Diplopoda & 0,009 & - \\
\hline Diptera (A) & 0,004 & - \\
\hline Diptera (L) & 0,146 & 35,499 \\
\hline Diptera $(\mathrm{P})$ & 0,068 & 1,007 \\
\hline Ephemeroptera $(\mathrm{N})$ & 0,138 & 3,881 \\
\hline Hemiptera & 0,198 & 1,353 \\
\hline Hymenoptera & 4,008 & 0,206 \\
\hline Lepidoptera terrestre $(\mathrm{L})$ & 0,027 & - \\
\hline Limacidae & 0,014 & - \\
\hline Material vegetal & 1,588 & 0,151 \\
\hline Megaloptera (N) & 0,002 & 0,016 \\
\hline Odonata $(\mathrm{N})$ & 1,026 & - \\
\hline Oligoqueta & 0,297 & 0,808 \\
\hline Ovos invertebrados & 0,245 & - \\
\hline Peixe & 42,859 & 5,021 \\
\hline Plecoptera $(\mathrm{N})$ & 0,005 & - \\
\hline Pyralidae (L) & 0,049 & 0,020 \\
\hline Sedimento & 0,709 & - \\
\hline Trichoptera (A) & 0,004 & - \\
\hline Trichoptera (L) & 0,092 & 48,955 \\
\hline Trichoptera $(\mathrm{P})$ & 0,006 & - \\
\hline
\end{tabular}

Tabela 2. Índices ecológicos e morfológico relacionados à alimentação natural de Rhamdia quelen (Rq) e Rhamdioglanis transfasciatus (Rt) ao longo do rio Macaé, RJ. S = riqueza de itens consumidos; $\mathrm{H}^{\prime}=$ amplitude de nicho; $\mathrm{PT}=$ posição trófica calculada; $\mathrm{Qi}=$ quociente intestinal; $\theta=$ sobreposição de nicho alimentar entre as duas espécies. Valores com asteriscos sinalizam índices que foram significativamente diferentes entre as espécies.

Table 2. Ecological and morphological indices related to the feeding of Rhamdia quelen $(\mathrm{Rq})$ and Rhamdioglanis transfasciatus (Rt) along the Macaé River, RJ. S = number of consumed items; $\mathrm{H}^{\prime}=$ niche breadth; $\mathrm{PT}=$ calculated trophic position; Q $i=$ intestinal coefficient; $\theta=$ niche overlap between the two species. Asterisks indicate indices that were significantly different between species.

\begin{tabular}{ccc}
\hline & Rq & Rt \\
\hline S & 53 & 41 \\
H' & $1,54^{*}$ & $1,36^{*}$ \\
PT & 2,65 & 2,52 \\
Qi & $0,81^{*}$ & $0,54^{*}$ \\
$\theta$ & & 0,51 \\
\cline { 2 - 3 }
\end{tabular}

Tabela 3. Índices ecológicos relacionados à alimentação natural de Rhamdia quelen $(\mathrm{Rq})$ e Rhamdioglanis transfasciatus (Rt) nas duas localidades de coocorrência das espécies no rio Macaé, RJ. $\mathrm{S}=$ riqueza de itens consumidos; H' = amplitude de nicho; $\mathrm{PT}=$ posição trófica calculada; $\mathrm{Q} i=$ quociente intestinal; $\theta=$ sobreposição de nicho alimentar entre as duas espécies.

Table 3. Ecological indices related to the feeding of Rhamdia quelen ( $\mathrm{Rq})$ and Rhamdioglanis transfasciatus (Rt) in the two sites of co-occurrence in the Macaé River, RJ. S = number of consumed items; H' = niche breadth; $\mathrm{PT}=$ calculated trophic position; $\theta=$ niche overlap between the two species. Asterisks indicate indices that were significantly different between species.

\begin{tabular}{lccccc}
\hline & \multicolumn{3}{c}{$\mathbf{R M 4}$} & & \multicolumn{3}{c}{ RM5 } \\
\cline { 2 - 3 } \cline { 5 - 6 } & $\mathbf{R q}$ & $\mathbf{R t}$ & & $\mathbf{R q}$ & $\mathbf{R t}$ \\
\hline S & 19 & 32 & & 46 & 25 \\
PT & 2,40 & 2,19 & & 2,44 & 2,28 \\
H' & 1,22 & 1,23 & & 1,50 & 1,59 \\
\cline { 2 - 3 } \cline { 5 - 6 } & \multicolumn{3}{c}{0,02} & & \multicolumn{3}{c}{0,06} \\
\hline
\end{tabular}

foi significativamente diferente entre as espécies. A posição trófica de $R$. quelen foi maior nas duas localidades de coleta. Os valores de S, H', PT e $\theta$ para as duas espécies nos locais de coocorrência estão relacionados na Tabela 3 .

\section{Discussão}

Ambas as espécies apresentam uma grande riqueza de itens alimentares em suas dietas. O consumo de diversos itens também foi relatado para $R$. quelen por Gomes et al. (2000) e Gomiero et al. (2007). Houve consumo predominante de recursos autóctones de origem bentônica para ambas as espécies, o que também foi observado para $R$. quelen por outros autores em riachos de Mata Atlântica (e.g. Casatti 2002). Embora R. quelen e R. transfasciatus tenham consumido muitos itens em comum, a proporção com que estes apareceram na dieta foi bastante diferente, havendo consumo de itens de maior porte por $R$. quelen, o que poderia ser função da diferença do tamanho de corpo (Zavala-Camim 1996) e/ou da boca (Wainwright \& Richard 1995), que podem restringir o tamanho da "presa". Entretanto, Casatti (2002), na bacia do alto rio Paraná, encontrou para $R$. quelen um consumo predominante de larvas de Trichoptera e Diptera, exatamente o que foi encontrado nesse estudo para $R$. transfasciatus. Este fato pode ser função do tamanho dos indivíduos de $R$. quelen, que foram, em média, cerca de $10 \mathrm{~cm}$ menores que os analisados no presente trabalho.

Classificamos $R$. quelen como carnívora e $R$. transfasciatus como invertívora, a última consumindo essencialmente insetos. Menezes et al. (2007) classificaram $R$. quelen da mesma forma, citando o consumo de insetos, crustáceos e pequenos peixes. Entretanto, Casatti (2002) classificou $R$. quelen como invertívora e Carneiro \& Mikos (2005), consideram a espécie onívora, indicando que isso facilita sua domesticação e condicionamento às condições de cultivo, pois favorece a aceitação do alimento artificial. O enquadradamento em diferentes categorias tróficas reflete o hábito generalista de $R$. quelen. Essa plasticidade é esperada, uma vez que a instabilidade natural dos sistemas lóticos favorece o desenvolvimento do generalismo alimentar (Lowe-McConnell 1999), comum em peixes neotropicais (Gerking 1994).

No rio Macaé, as proporções de itens de origem vegetal consumidas foram muito pequenas para que as espécies fossem consideradas onívoras (Zavala-Camin 1996). Esse baixo consumo sugere ingestão acidental do material vegetal, também observado por Sabino \& Castro (1990) para um Characiforme em riacho de Mata Atlântica. Os quocientes intestinais médios (Qi) reforçam a hipótese de ingestão acidental de plantas, corroborando a classificação trófica estabelecida para ambas as espécies. De fato, valores de Qi podem contribuir para a classificação de espécies em categorias tróficas amplas (Angelescu 


\begin{tabular}{|l|}
\hline 1. Lepidoptera terrestre \\
2. Limacidae \\
3. Ovos de invertebrados \\
4. Plecoptera (ninfa) \\
5. Peixes \\
6. Decapoda \\
7. Trichoptera (adulto) \\
8. Coleoptera \\
9. Hymenoptera \\
10. Oligochaeta \\
11.Caelifera \\
12. Aracnidae \\
13. Megaloptera (ninfa) \\
14. Alga Filamentosa \\
15. Diplópode \\
16. Trichoptera (pupa) \\
17. Diptera (adulto) \\
18. Pyralidae (larva) \\
19. Diptera (pupa) \\
20. Odonata (ninfa) \\
21. Trichoptera (larva) \\
22. Hemiptera \\
23. Ephemeroptera (ninfa) \\
24. Diptera (larva) \\
25. Material vegetal \\
\hline
\end{tabular}
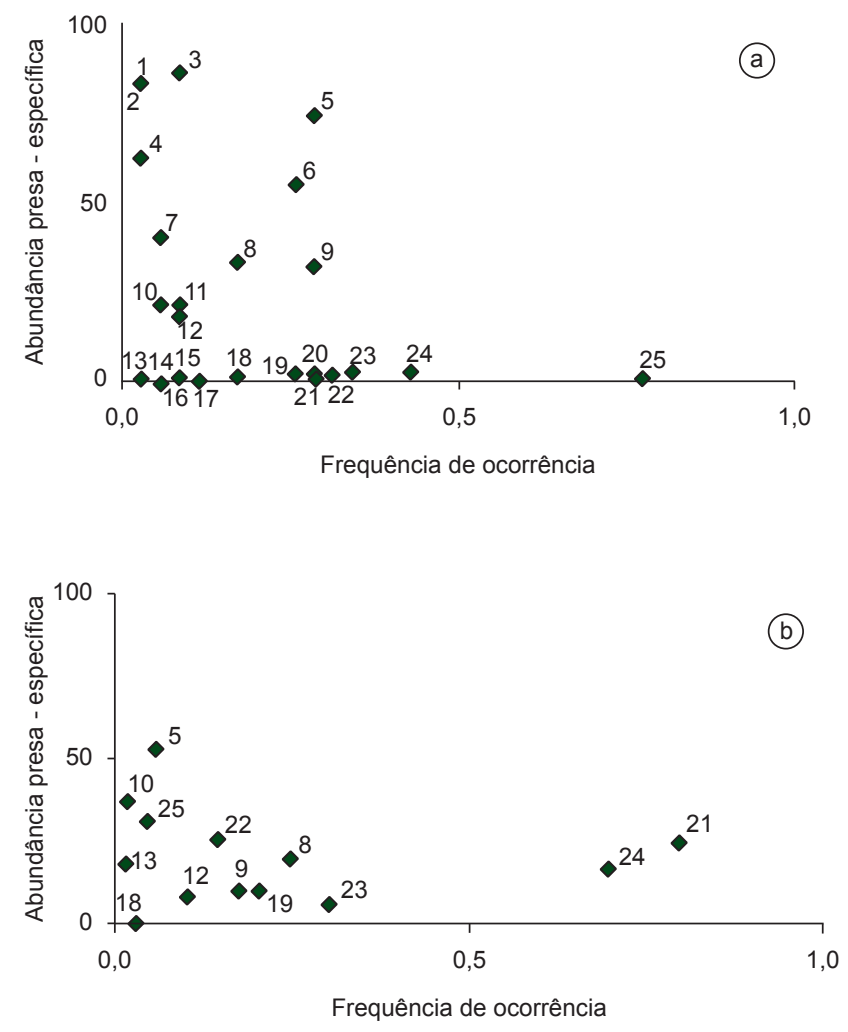

Figura 2. Diagrama de Amundsen et al. (1996) para representação da estratégia alimentar das populações de a) $R$. quelen e b) $R$. transfasciatus no rio Macaé, RJ.

Figure 2. Amundsen diagram for the representation of feeding strategies of populations of a) R. quelen and b) R. transfasciatus in the Macaé River, RJ.

\& Gneri 1949), porém, não seriam suficientes para predizer o grau de especialização dentro de uma determinada dieta (Albrecht et al. 2001). Pode-se extrapolar um padrão a partir de estudos baseados em espécies remotamente relacionadas (Barbieri et al. 1994) ou espécies da mesma família (Fryer \& Iles 1972), como é o caso desse trabalho. De acordo com esse padrão, altos valores de Qi podem agrupar espécies herbívoras, enquanto valores intermediários são associados com os onívoros e baixos valores com carnívoros e piscívoros (Albrecht et al. 2001), sendo as duas espécies desse estudo encaixadas na última classificação.

Da mesma forma, a posição trófica calculada, tanto considerando as dietas totais quanto as dietas nos locais de coocorrência, complementa os resultados anteriores, indicando que ambas as espécies são consumidoras secundárias, com valores um pouco maiores para $R$. quelen. Mesmo não compartilhando os mesmos táxons de presas, pertencem ao mesmo nível trófico, exercendo provavelmente papéis semelhantes nos processos de fluxo de energia no ecossistema. Porém, esse cálculo de posição trófica assume que as frações consumidas refletem a contribuição energética relativa de cada item para o consumidor, o que não necessariamente reflete a realidade, pois os recursos podem ser assimilados em proporções diferentes daquelas observadas com a análise da dieta, que é apenas um "retrato" do que foi consumido em determinado momento (Williams \& Martinez 2004). Isso evoca a necessidade de uma análise complementar, por exemplo, utilizando isótopos estáveis. Por outro lado, embora o fluxo de energia seja central para o entendimento do funcionamento do ecossistema, pode ser inapropriado se o foco é a comunidade e a dinâmica das populações, em parte porque o fluxo de energia e produção de biomassa são propriedades emergentes das interações dentro da teia (Polis \& Strong 1996).

Considerando a dieta das duas espécies ao longo do rio Macaé, $R$. quelen apresentou amplitude de nicho um pouco maior do que
R. transfasciatus, em função da maior variedade de itens consumidos. A amplitude do nicho não foi diferente entre as espécies em cada localidade, sugerindo que a capacidade de explorar diferentes itens disponíveis no ambiente e, consequentemente, a plasticidade trófica, seja equivalente. Isso se confirma ao considerarmos a riqueza de itens consumidos na dieta como um todo e em RM5.

A composição qualitativa muito semelhante na dieta de $R$. quelen e $R$. transfasciatus, mesmo que as quantidades de consumo tenham sido diferentes, se refletiu no valor de sobreposição de nicho, considerando a dieta ao longo do rio Macaé. Porém, ao considerar apenas os locais de coocorrência, as diferenças se acentuam e essa similaridade torna-se quase nula, demonstrando a ocorrência de partilha de recursos alimentares em nível local. Isso permite aceitar a hipótese de que a simpatria dessas duas espécies é possível devido a alguma diferenciação ecológica na dieta. Essa diferenciação pode ser fruto de exposição a disponibilidades diferentes de recursos alimentares, consequência de segregação espacial, uma vez que, de acordo com os dados de captura no Rio Macaé (Brito 2007) e da literatura, R. quelen é uma espécie bentônica que demonstra preferência por remansos e águas profundas com fundo de folhiço e matacões (Barreto \& Aranha $2005)$ e $R$. transfasciatus é típica de corredeiras e ambientes pedregosos (Menezes et al. 2007). Ou seja, se tomarmos cada localidade como ponto de referência, não se percebe segregação espacial (as espécies co-ocorrem em RM4 e RM5) e a diferenciação ecológica se restringe à utilização de diferentes itens na dieta (segregação trófica), como é esperado para ambientes aquáticos (Ross 1986). Porém, analisando o meso-hábitat (remanso e canal), há diferenças nos locais ocupados, podendo ser este um dos causadores da divergência alimentar. A segregação espacial já foi descrita em outros trabalhos como um fator que evita a competição e permite a coexistência de espécies (e.g. Agostinho et al. 2003, Winemiller 1991). Winemiller 
\& Kelso-Winemiller (1994), estudando duas espécies de piscívoros no rio Zambezi na África, encontraram que a potencial competição não se realizava devido à segregação de hábitats e ao consumo de presas em proporções diferentes, o mesmo encontrado para $R$. quelen e $R$. transfasciatus nesse trabalho. A ocupação de diferentes hábitats representa uma exposição a condições diferentes de disponibilidade de recursos, determinando os itens a serem consumidos pelas espécies. Nesse sentido, Owen \& Karr (1978) e Fausch (1984) sugerem que a especialização de hábitat é um fator essencial para a estrutura de comunidades de peixes de riachos, enquanto que a especialização alimentar dependeria da disponibilidade de recursos. Essas considerações, somadas ao que foi encontrado para as espécies no presente estudo, reforçam a importância de considerarmos a escala espacial em estudos ecológicos, pois um padrão que é aparente em determinada escala pode não ser em uma escala menor, uma vez que diferentes fatores podem influenciar o uso de hábitat por uma espécie nas diferentes escalas espaciais (Woodward \& Hildrew 2002).

Além da exploração de hábitats diferentes, a estratégia alimentar utilizada pelas populações por si só já é um fator que diferencia as espécies ecologicamente (Costello 1990, Amundsen et al. 1996). Como se esperaria, não há item dominante (consumido em grande quantidade pela maioria da população) na dieta de ambas as espécies estudadas, reforçando os resultados que indicam seu generalismo alimentar. Entretanto, é possível identificar diferenças na estratégia alimentar de cada uma. Segundo Bolnick (2003), uma população pode ser generalista de duas formas: a primeira, quando a população é formada por grupos de especialistas, havendo grande variação individual (alto componente interfenótipo); a segunda, quando todos, ou a maior parte, dos indivíduos que compõem a população são, eles mesmos, generalistas (alto componente intrafenótipo). Analisando essas duas formas como extremos de um gradiente (Futuyma \& Moreno 1988), as duas espécies estariam em um meio termo, sendo que $R$. quelen estaria mais próxima da primeira descrição e $R$. transfasciatus estaria mais próxima do outro extremo. Rhamdia quelen apresenta maior contribuição do componente interfenótipo, indicando a presença de especialização de grupos de indivíduos dentro da população. Apenas Material Vegetal foi consumido por mais da metade da população, porém em quantidades extremamente baixas. Na população de $R$. transfasciatus a maior parte dos indivíduos se comporta de forma generalista. Apenas o item Peixe foi consumido em grande quantidade por uma parcela restrita da população, enquanto que os itens Larva de Diptera e de Trichoptera, foram consumidos pela maior parte da população, contribuindo para o componente intrafenótipo da largura do nicho, de acordo com os valores do Índice Alimentar. Embora não muito comum nos estudos de ecologia alimentar, a existência de especialização individual vem sendo abordada em diversos estudos como a possibilidade de ser uma estratégia contra a competição intraespecífica (Fretwell 1969, Bolnick et al. 2002, Bolnick et al. 2003, Sargeant 2007), incrementando o potencial de sucesso da espécie. Dessa forma, a utilização de recursos diferentes entre os grupos de indivíduos pode significar uma disponibilidade menor dos recursos utilizados por $R$. quelen, impondo um comportamento que promove maior diferenciação individual.

As espécies Rhamdia quelen e Rhamdioglanis transfasciatus apresentaram nicho alimentar semelhante, mas com sobreposição quase nula nos locais de coocorrência, indicando a existência de partilha de recursos. Embora ocupando posições tróficas equivalentes, $R$. quelen e $R$. transfasciatus não seriam espécies redundantes ecologicamente, pois apresentam distinção em outros aspectos alimentares, tal como o micro-hábitat utilizado, reforçando que estudos sobre ecologia trófica devem ampliar-se além da caracterização da dieta das espécies.

\section{Agradecimentos}

O edital universal CNPq financiou o projeto 479257/2004-3, dentro do qual o presente estudo foi realizado. MPA agradece ao CNPq pela bolsa PDJ (processo n. 151148/2005-8). Somos gratas a toda a equipe do Laboratório de Ecologia de Peixes da UFRJ que participou das coletas, a Marcelo F. G. Brito pelo fornecimento dos dados relativos ao Rio Macaé e distribuição das espécies, e a Érica P. Caramaschi e Juliana R. Pereira pela revisão crítica da versão final do manuscrito.

\section{Referências Bibliográficas}

ABELHA, M.C.F., AGOSTINHO A.A. \& GOULART, E. 2001. Plasticidade trófica em peixes de água doce. Acta Sci. 23(42):425-434.

ADAMS, S.M., KIMMEL, B.L. \& PLOSKEY, G.R. 1983. Sources of organic matter for reservoir fish production: a trophic-dynamics analysis. Can. J. Fish Aquat. Sci. 40:1480-1495.

AGOSTINHO, C.S., HAHN, N.S. \& MARQUES, E.E. 2003. Patterns of food resources use by two congeneric species of piranhas (Serrasalmus) on the upper Paraná river floodplain. Braz. J. Biol. 63(2):177-182.

ALBRECHT, M.P., FERREIRA, M.F.N. \& CARAMASCHI, E.P. 2001. Anatomical features and histology of the digestive tract related neotropical omnivorous fishes (Characiformes; Anostomidae). J. Fish Biol. 58:419-430.

ALBRECHT, M.P. \& CARAMASCHI, E.P. 2003. Feeding ecology of Leporinus friderici (Teleostei; Anostomidae) in the upper Tocantins river, central Brazil, before and after installation of a hydroelectric plant. Stud. Neotrop. Fauna E. 38(1):33-40.

ALBRECHT, M.P. 2005. Estrutura trófica da ictiofauna do rio Tocantins na região sob influência as usina hidrelétrica Serra da Mesa, Brasil Central. Tese de Doutorado, Universidade Federal do Rio de Janeiro, Rio de Janeiro. 194 p.

ALMEIDA, V.L.L. 1994. Utilização de recursos alimentares por peixes piscívoros da planície de inundação do alto rio Paraná $\left(22^{\circ} 40^{\prime}-22^{\circ}\right.$ 50'S $/ 53^{\circ} 40^{\prime}$ W) Brasil. Dissertação de Mestrado, Universidade Estadual de Maringá, Maringá.

AMARAL, K.J. 2003. Estuário do Rio Macaé: modelagem computacional como ferramenta para o gerenciamento integrado de recursos hídricos. Rio de Janeiro.

AMUNDSEN, P.A., GABLER, H.M. \& STALDVIK, F.J. 1996. A new approach to graphical analysis of feeding strategy from stomach contents data: modification of the Costello (1990) method. J. Fish Biol. 48:607-614.

ANDRIAN, I.F., DÓRIA, C.R.C., TORRENTE, G. \& FERRETI, C.M. 1994. Espectro alimentar e similaridade na composição da dieta de quatro espécies de Leporinus (Characiformes, Anostomidae) do rio Paraná, Brasil. Rev. UNIMAR. 16(3):97-106.

ANDRIAN, I.F. \& BARBIERI, G. 1996. Espectro alimentar e variações sazonal e espacial na composição da dieta de Parauchenipterus galeatus Linnaeus, 1766, (Siluriformes, Auchenipteridae) na região do reservatório de Itaipu, PR. Rev. Bras. Biol. 56(2):409-422.

ANGELESCU, V. \& GNERI, F. 1949. Adaptaciones del aparato digestivo al regimes alimenticios en algunos peces del Rio Uruguay y del Rio de La Plata. Revista del Instituto Nacional de Ciencias Naturales. 1:161-261.

BARBIERI, G., PERET, A.C. \& VERANI, J.R. 1994. Notas sobre a adaptação do trato digestivo ao regime alimentar em espécies de peixes da região de São Carlos (SP). I Quociente intestinal. Rev. Bras. Biol. 54:63-69.

BARRETO, A.P. \& ARANHA, J.M.R. 2005. Assembléia de peixes de um riacho da Floresta Atlântica: composição e distribuição espacial (Guaraqueçaba, Paraná, Brasil). Acta Sci. 27(2): 153-160.

BAPTISTA, D.F., DORVILLÉ, L.F.M., NESSIMIAN, J.L. 2001. Spatial and temporal organization of aquatic insects assemblages in the longitudinal gradient of a tropical river. Rev. Bras. Biol. 61(2):295-304.

BIZERRIL, C.R.S.F. \& PRIMO, P.B. 2001. Peixes de águas interiores do Estado do Rio de Janeiro. GTZ/SEMADS, Rio de Janeiro.

BOCKMANN, F. \& GUAZZELLI, A.Y.G.M. 2003. Heptapteridae. In Check list of the freshwater fishes of South and Central America. (R.E. Reis, S.O. Kullander \& C.J Ferraris Jr., orgs). EDIPUCRS, Porto Alegre, p. 406-431.

BOLNICK, D.I., YANG, L.H., FORDYCE, J.A., DAVIS, J.M. \& SVANBÄCK, R. 2002. Measuring individual-level resource specialization. Ecology. 83:2936-2941. 
BOLNICK, D.I., SVANBÄCK, R., FORDYCE J.A., YANG L.H., DAVIS J.M., HULSEY C.D. \& FORISTER M.L. 2003. The ecology of individuals: incidence and implications of individual specialization. Am. Nat. 161:1-20.

BORROR, D.J. \& DELONG, D.M. 1969. Introdução ao estudo dos insetos. Blucher/USP/EDUSP, São Paulo.

BORROR, D.J., TRIPLEHORN, C.H. \& JOHNSON, N.F. 1992. An introduction to the study of the insects. 6 ed. Saunders College Publishing, Florida.

BRITO, M.F.G. 2007. Atividade reprodutiva dos peixes do rio Macaé (RJ) em função do gradiente longitudinal. Tese de Doutorado, Universidade Federal do Rio de Janeiro, Rio de Janeiro. 168 p.

CARNEIRO, P.C.F. \& MIKOS, J.D. 2005. Freqüência alimentar e crescimento de alevinos de jundiá Rhamdia quelen. Cienc. Rural. 35(1):187-191.

CASATTI, L. 2002. Alimentação dos peixes em um riacho do Parque Estadual Morro do Diabo, bacia do alto rio Paraná, sudeste do Brasil. Biota Neotrop. 2(2):http:// www.biotaneotropica.org.br/v2n2/pt/abstract?arti cle+BN02502022002.

CORTÉS, E. 1997. A critical review of methods of studying fish feeding based on analysis of stomach contents: application to elasmobranch fishes. Can. J. Fish. Aquat. Sci. 54:726-738.

COSTELLO, M.J. 1990. Predator feeding strategy and prey importance: a new graphical analysis. J. Fish Biol. 36:261-263.

FAUSCH, K.D. 1984. Profitable stream positions for salmonids: relating specific growth rate to net energy gain. Can. J. Zool. 62:441-451.

FRETWELL, S.D. 1969. Dominance behavior and winter habitat distribution in juncos (Junco hyemalis). Bird-Band. 40:1-25.

FRYER, G. \& ILES, T.D. 1972. The cichlid fishes of the great lakes of Africa: their biology and evolution. Oliver \& Boyd, Edinburgh.

FUGI, R. \& HAHN, N.S. 1991. Espectro alimentar e relações morfológicas com o aparelho digestivo de três espécies de peixes comedores de fundo do rio Paraná, Brasil. Rev. Bras. Biol. 51 (4):873-879.

FUTUYMA, D.J. \& MORENO, G. 1988. The evolution of ecological specialization. Annu. Rev. Ecol. Syst. 19:207-233

GERKING, S.D. 1994. Feeding ecology of fish. Academic Press, California.

GOMES, L.C., GOLOMBIESKI, J.I., CHIPPARI-GOMES, A.R. \& BALDISSEROTTO, B. 2000. Biology of Rhamdia quelen (Teleostei, Pemelodidae). Cienc. Rural. 30(1):179-185.

GOMIERO, L.M., SOUZA, U.P. \& BRAGA, F.M.S. 2007. Reprodução e alimentação de Rhamdia quelen (Quoy \& Gaimard, 1824) em rios do Núcleo Santa Virgínia, Parque Estadual da Serra do Mar, São Paulo, SP. Biota Neotrop. 7(3):http:// www.biotaneotropica.org.br/v7n3/pt/abstrac t?article+bn01907032007.

HERDER, F. \& FREYHOF, J. 2006. Resource partitioning in a tropical stream fish assemblage. J. Fish Biol. 69:571-589.

HUTCHINSON, G.E. 1957. Concluding remarks: cold spring harbor symp. Quant. Biol. 22:415-427

HYNES, H.B.N. 1950. The food of fresh-water sticklebacks (Gasterosteus aculeatus and Pygosteus pungitius), with a review of methods used in studies of the food of fishes. J. Anim. Ecol. 19:36-57.

HYSLOP, E.J. 1980. Stomach contents analysis: a review of methods and their application. J. Fish Biol. 17:411-429.

KAWAKAMI, E. \& VAZZOLER, G. 1980. Método gráfico e estimativa de índice alimentar aplicado no estudo de alimentação de peixes. Boletim do Instituto Oceanográfico de São Paulo. 29(2):205-207.

KRAMER, D.L. \& BRYANT, M.J. 1995. Intestine lenght in the fishes of a tropical stream 2. Relationships to diet: the long and short of a convoluted issue. Environ. Biol. Fish. 42:129-141

KREBS, C.J. 1999. Ecological methodology. Addison Wesley Longman, New York.

LOREAU, M. 2000. Biodiversity and ecosystem functioning: recent theoretical advances. Oikos. 91(1):3-17

LOUREIRO, V.E. \& HAHN, N.S. 1996. Dieta e atividade alimentar da traíra, Hoplias malabaricus (Bloch, 1794) (Osteichthyes, Erythrinidae), nos primeiros anos de formação do reservatório de Segredo-PR. Acta Limnol. Bras. 8:195-205.

LOWE-MCCONNELL, R.H. 1987. Ecological studies in tropical fish communities. Cambridge University Press, Cambridge.

LOWE-MCCONNELL, R.H. 1999. Estudos ecológicos em comunidades de peixes tropicais (A.E.A.M. Vazzoler, A.A. Agostinho \& P.T.M. Cunnhingham, trads.). EDUSP, São Paulo.

MENEZES, N.A., WEITZMAN, S.H., OYAKAWA, O.T., LIMA, F.C.T., CASTRO, R.M.C. \& WEITZMAN, M.J. 2007. Peixes de água doce da Mata Atlântica: lista preliminar das espécies e comentários sobre a conservação dos peixes de água doce neotropicais. Museu de Zoologia da Universidade de São Paulo, São Paulo.

MERRIT, R.W. \& CUMMINS, K.W. 1996. Introduction to the aquatic insects of North America. Kendall/Hunt Pub. Co., Iowa.

MICHELETTI, C.V. \& UIEDA, V.S. 1996. Food resources partitioning among Sciaenid fishes (Perciformes: Sciaenidae) of the Flamengo bay, Ubatuba, southeastern Brazil. Arq. Biol. Tecnol. 39(3):639-649.

PIANKA, E.R. 1973. The structure of lizard communities. Annu. Rev. Ecol. Syst. 4:53-74.

POLIS, G.A. \& STRONG, D.R. 1996. Food web commplexity and community dynamics. Am. Nat. 147:813-846.

OWEN, T.G. \& KARR, J.R. 1978. Habitat structure and stream fish communities. Ecology. 4:199-215.

RESETARITS, W.J. \& CHALCRAFT, D.R. 2007. Functional diversity within a morphologically conservative genus of predators: implications for functional equivalence and redundancy in ecological communities. Funct. Ecol. 21:793-804.

ROSS, S.T. 1986. Resource partitioning in fish assemblages: a review of field studies. Copeia (2):352-388.

SABINO, J. \& CASTRO, R.M.C. 1990. Alimentação, período de atividade e distribuição espacial dos peixes de um riacho da floresta Atlântica (Sudeste do Brasil). Rev. Brasil. Biol. 50:23-36.

SARGEANT, B.L. 2007. Individual foraging specialization: niche width versus niche overlap. Oikos. 116:1431-1437.

Secretaria de Estado de Meio Ambiente e Desenvolvimento Sustentável - SEMADS. 2001. Bacias hidrográficas e rios fluminenses: síntese informativa por macrorregião ambiental. Rio de Janeiro. 73 p.

SHOENER, T.W. 1974. Resource portioning in ecological communities. Science. 185:27-39.

WAINWRIGHT, P.C. \& RICHARD, B.A. 1995. Predicting patterns of prey use from morphology of fishes. Environ. Biol. Fish. 44:97-113.

WEATHERLEY, A.H. 1963. Notions of niche and competition among animals, with special reference to freshwater fish. Nature. 197(5):14-17.

WILLIAMS, R.J. \& MARTINEZ, N.D. 2004. Limits to trophic levels and omnivory in complex food webs: theory and data. Am. Nat. 163(3):458-468.

WINEMILLER, K.O. 1991. Comparative ecology of Serranochromis species (teleostei: Ciclhidae) in the Upper Zambezi River. J. Fish Biol. 39:617-639.

WINEMILLER, K.O. \& KELSO-WINEMILLER, L.C. 1994. Comparative ecology of the African pike, Hepsetus odoe, and tigerfish, Hydrocynus forskahlii, in the Zambezi River floodplain. J. Fish Biol. 45:211-225.

WOODWARD, G. \& HILDREW, A.G. 2002. Food web structure in riverine landscapes. Freshwat. Biol. 47:777-798.

WOOTTON, R.J. 1990. Ecology of teleost fishes. Chapman \& Hall, New York.

ZAR, J.H. 1984. Biostatistical analysis. Prentice-Hall, New Jersey.

ZAVALA-CAMIN, L.A. 1996. Introdução aos estudos sobre alimentação natural em peixes. EDUEM, Maringá. 